\title{
Development of cylindrical stepwedge phantom for routine quality controls of a helical tomotherapy machine
}

\author{
Krzysztof Mikołajczyk ${ }^{a, *}$, Tomasz Piotrowski ${ }^{b}$
}

\author{
a Department of Medical Physics, Greater Poland Cancer Centre, 15th Garbary Street, 61-866 Poznań, Poland \\ ${ }^{\mathrm{b}}$ Department of Electroradiology, University of Medical Sciences, 10 Fredry Street, 61-701 Poznań, Poland
}

Received 25 August 2011; received in revised form 25 October 2011; accepted 11 December 2011

Available online 30 December 2011

\author{
KEYWORDS \\ Cylindrical stepwedge \\ phantom; \\ QA; \\ Tomotherapy; \\ Parameter \\ verification
}

\begin{abstract}
The aim of this study was to design a cylindrical stepwedge phantom and an appropriate treatment procedure, based on which parameters of tomotherapy machine and generated beam of radiation will be defined. The accuracy of parameter determination, which can be defined with the aid of the measurement system, was also evaluated.

The cylindrical phantom that we developed and manufactured (stepwedge phantom) consists of four cylinders with different diameters made of polycaprolactam-PA-6, i.e. material with high mechanical strength, low water absorption (making measurements repeatable) and a density comparable to that of human soft tissues. The appropriate treatment procedure is carried out in a dynamic mode, which is focused on specific properties of the tomotherapy machine. It means that a phantom situated on the couch moves to the inside of the rotating linear accelerator.

A total of 18 procedures were implemented in order to calculate the following parameters: couch velocity, dose rate value at a depth, Dose Ratio coefficients, dose variation (so-called Dose Flatness) coefficients, and the time of gantry rotation. Reference intervals for these parameters were determined to be as follows: for the couch velocity: $\pm 1.2 \%$, the average dose rate measured at depth: $\pm 1.8 \%$, the calculated values of the coefficients Dose Ratio: $\pm 0.5 \%$ and Dose Flatness: $(0.53-0.65) \%$, the time of gantry rotation: $\pm 3 \%$.

The final results showed that during a single irradiation procedure, which lasts $5 \mathrm{~min}$, the cylindrical stepwedge phantom allows to precisely determine the values of the abovementioned parameters. Its use in the daily dosimetric measurements can ensure better control of the work of the tomotherapy machine.
\end{abstract}

(c) 2011 Associazione Italiana di Fisica Medica. Published by Elsevier Ltd. All rights reserved.

\footnotetext{
* Corresponding author.

E-mail address: krzysztof.mikolajczyk@gmx.com (K. Mikołajczyk).
} 


\section{Introduction}

In teletherapy, quality assurance involves performing a number of procedures that test radiotherapy equipment in terms of proper mechanical work as well as radiation dosimetry. A number of tests have been developed for tomotherapy machines [1-4] that partly overlap the tests for traditional linear accelerators [5-10]. Depending on the movements of the linac (mounted on the ring gantry) and the couch (while treating or while measuring) the appropriate tests can be: static, where the gantry and the couch are fixed, or irradiation is performed from a few selected angles; and dynamic, where the couch is in motion while the gantry is rotating. In general, all tests can be divided into five groups. Most of these tests require additional equipment and accessories. Beside megavoltage computed tomography (MVCT) detector, which is part of the tomotherapy equipment, the system includes thimble ionization chambers (Exradin A17 and A1SL ion chambers), X-ray films, phantoms: Water Tank, Slab Solid Water Phantom, Cheese Phantom (some of them supplied by the vendor).

The first group of tests allows for the verification of several mechanical alignments. This set of tests check: the alignment of the radiation source in the $x$-direction against multi-leaf collimator (MLC), in the $y$-direction against the $y$ jaw, jaw symmetry, beam planarity (to verify that the beam is parallel to the plane of gantry rotation), the lateral alignment of the MLC relative to the center of rotation and that the MLC is aligned parallel to the rotational plane, MVCT detector alignment (ensures that the MVCT detector array is centered and aligned with respect to the jaws) $[2,11]$. All tests are performed in static mode. On-board MVCT detector and film (Kodak EDR2 Film) placed between two solid water plates are used in these tests. The last time a new independent tool for tomotherapy QA was presented [12]. The dose magnifying glass (DMG) is a 128 channel array of Si strip detectors. It was used to measure three tomotherapy QA parameters: MLC alignment, leaf latency and leaf output factor (LOF).

The second group of tests checks the MVCT detector: monitoring image quality (spatial resolution and contrast, noise, uniformity), measuring the dose delivered during acquisition of MVCT and verifying the correctness of conversion density - HU. MVCT detector and Cheese Phantom are required in this case [11]. The third group of tests allows for the verification of laser alignment, the red laser movement, the Virtual Isocenter and movement, the longitudinal motion alignment and the sag of treatment couch [11].

Tomotherapy is unique in its action, due to the irradiation provided in a helicoidally way and the behavior of the MLC collimator [13]. The fourth group of tests allows for the verification of the uniformity of the couch motion, the leaf opening synchronized with gantry rotation and the couch translation synchronized with gantry rotation [14]. Slab Solid Water Phantom and films are required in these tests.

The last group of tests allows for the verification of the beam quality. These includes the measurements of longitudinal, transversal and percentage depth dose (PDD) profiles. Water Tank and ionization chamber are used in these tests [11]. The MVCT detector can be used for measuring the transversal profile [15]. The next test in this group monitors the output consistency (stationary and rotational output) using an ion chamber or on-board monitor chambers (the detectors of tomotherapy machines). The measurement of rotational output variation can be performed using the Cheese Phantom and films [14] or monitor chambers [11]. The last test is a dosimetric verification which allows us to compare the dose to be delivered by the treatment planning system (TPS) with the measured, for the plan using intensity modulated radiation therapy (IMRT) [11,16-18].

The last time the Tomotherapy Inc. has offered TQA (TomoTherapy Quality Assurance ${ }^{\mathrm{TM}}$ ). This easy-to-use application automates the collection - and simplifies the analysis - of key metrics for machine QA. It is intended to monitor the changes in the system performance that may provide early indications for maintenance or dosimetric validation. Output data is acquired only from the dose monitoring system and the detector array. It allows to measure the monitor chamber and detector output constancy, verify the rotational variation and by using the stepwedge to provide a measurement of the couch velocity, energy, and synchrony of the couch, the multi-leaf collimator and the gantry.

Large variety of tests ensures proper operation of equipment. However, they are time-consuming and the amount of time used for patient treatment is significantly reduced. The solution in this case seems to be the TQA. It allows us to provide measurements of several parameters of the device at the same time. However, the TQA and most of the verification tests use on-board equipment like MVCT detectors or ionization chambers. There are few advanced tests performed independently of the irradiation machine.

All of the above deficiencies in the verification tests have contributed to the design of the phantom - the cylindrical stepwedge phantom. The measurement system allows us to verify the compliance in several areas of machine work, in a single irradiation procedure: the couch velocity, the gantry time rotation and changes in the dose rate in the longitudinal plane. An additional requirement was that the measurements were made independently from the machine, so that the results could be more objective by using of additional equipment.

\section{Material and methods}

\section{The preparation of cylindrical stepwedge phantom}

The phantom used was made of cast polyamide PA-6 (polycaprolactam). This material is fully suitable for dosimetric applications of this study. Its stiffness and hardness make it possible, as a result of further processing, to obtain the desired shape with specific dimensions. Mechanical resistance guarantees the use of the material without fear of any damage caused by everyday use, and low water absorption, compared with other plastics, ensures constancy of internal environment of the measuring system and thus the repeatability of measurements. Density of $1.12 \mathrm{~g} / \mathrm{cm}^{3}$ is comparable to that of human soft tissues.

The phantom consists of four coaxially connected cylinders with diameters of $40 \mathrm{~mm}, 60 \mathrm{~mm}, 80 \mathrm{~mm}, 100 \mathrm{~mm}$ 
and length of $40 \mathrm{~mm}$ each (Fig. 1). Through the center of the phantom runs a hole with a diameter of $10.5 \mathrm{~mm}$. All dimensions have the tolerance of $0.15 \%$, resulting from elasticity of the material, increased coefficient of thermal expansion and water absorbability during the processing of elements. The shape of the phantom has three main advantages:

(a) construction based on the cylinder allows to carry out treatment procedures in dynamic mode, and thus takes into account the specific characteristics of tomotherapy machine,

(b) four different diameters of the cylinders allow to perform measurements at different depths of the phantom during one test procedure, without the need for additional interference in the system during the measurements,

(c) combination of cylindrical elements allows to analyze the performance of the machine and the beam on the environment boundary of different thicknesses.

\section{Designing a measurement system}

The design of the measuring system is another important element that allows to use the phantom in practice. Fig. 2 shows schematically various elements of the system and their arrangement.

In the central part of the system, there is the ring gantry of the tomotherapy machine (marked: 1) and the patient couch (marked: 2 ), which moves along the $y$-axis during the irradiation procedure. The tripod phantom (marked: 4) is placed on the couch in such a way that the entire volume of the phantom could be off the couch. With this solution, the measurement of dose in the phantom will not be inappropriate with an error arising from the absorption and scattering of radiation passing through the couch. The ionization chamber (marked: 5) is a key element of the system serving as a measuring instrument. It remains suspended motionless in the isocentre of the tomotherapy machine during the whole procedure of irradiation. The

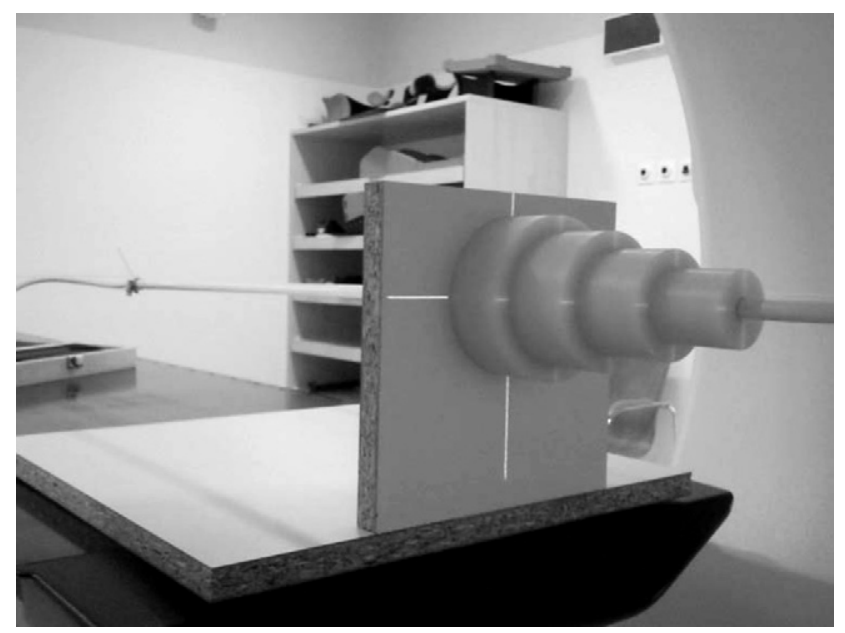

Figure 1 Picture of the phantom made of polyamide PA-6 and the measuring system. phantom (marked: 3 ) then moves to the inside of the ring gantry, along the $y$-axis. The system uses a rigid, plastic (PVC) tube with a wall thickness of $2 \mathrm{~mm}$ (marked: 6), which also separates the ionization chamber, with a diameter of $6.5 \mathrm{~mm}$, from the movable phantom and ensures the stability of the chamber during treatment procedures. The system to fix the measuring system (marked: 9), tube clamps (marked: 7) and cord clamping (marked: 8) are required for the immobilization of the tube. They provide regular, fixed arrangement of the chamber during dosimetric measurements.

Measurements of radiation dose were made using the Exradin A1SL ionization chamber. The chamber is connected to the electrometer TomoElectrometer, which is connected to the PC. TomoTherapy ${ }^{\circledR}$ Electrometer Software Measurment System (TEMS) allows for data acquisition and control of the electrometer to be done from the computer. Measurements are made at a voltage of $300 \mathrm{~V}$ in the chamber and sampling frequency signal of $100 \mathrm{~ms}$.

\section{Calibration of the measuring system}

Before measurements, the measuring system has to be properly calibrated. The main part of the calibration phantom is to determine the relative position of the virtual and actual isocentre of the machine, and to ensure the correct placement of the ionization chamber. Placed on the tripod were special tags to allow reference to the tomotherapy machine position of the phantom. Before each irradiation, their location must coincide with the laser system in the room. These markers help re-arrange the measuring system in the consecutive fractions of radiotherapy.

\section{Designing test procedure}

The aim of the procedure was to irradiate the phantom in dynamic mode, with a certain width of collimator jaws and Pitch Factor, without the use of beam intensity modulation. During the procedure the ionization chamber is placed in the isocentre of the tomotherapy machine and the phantom with tripod moves to the inside of the ring gantry. The linac mounted on the ring gantry is still rotating.

This procedure was implemented by using the Create Procedure function of the TomoTherapy Operator Station software. The parameter settings for the procedure are presented in Table 1. During the whole process of radiation, the system collects information about the charge accumulated in the ionization chamber placed inside the phantom. This data was converted to dose rate expressed in gray per second.

\section{Signal analysis}

The signal which is derived from data acquired from the electrometer is shown Fig. 3. It illustrates the dose rate changes in time domain. Each section (part 1, 3, 5 and 7) of the received signal corresponds to the dose rate measured inside the phantom for each cylinder. The primary signal in these areas is not perfectly flat, but fluctuates around the average (mean dose rate at the depth) with a characteristic 


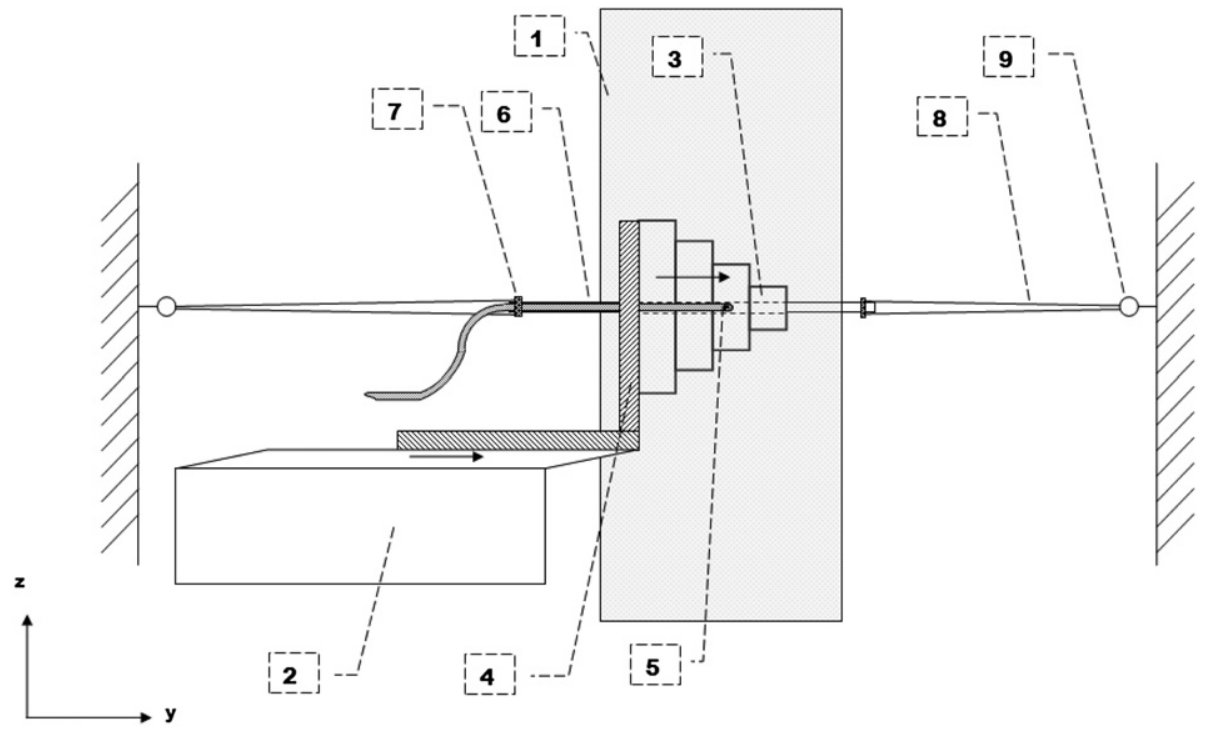

Figure 2 Diagram of the measuring system. View in the plane yz. For description of individual system components see the text. Diagram is not to scale.

frequency. This periodic change in the signal, known as Variation of Rotation, is associated with rotation of linac mounted on the ring gantry. Parts between successive sections (part 2, 4 and 6) are of equal length and are inclined at an angle. These areas correspond to the transition moment at which the ionization chamber is located on the border of two cylinders of the phantom. Parameters of all these sections were used to determine parameters of the tomotherapy machine and the generated beam.

For the purpose of a rapid, preliminary analysis of the received signal, a macro stepwedge Analysis was created. It is written in a programming language based on Visual Basic and implemented in a Microsoft Excel spreadsheet. The macro automates some processes which must be performed for each signal. It consists of several blocks based on different algorithms.

The raw data is transferred from TEMS to a spreadsheet. The first is to identify the proper signal. Each sample of signal is compared with a noise threshold, specified in the parameters of the program, and if it exceeds the set value, it is transferred for further analysis. The next step is to execute a series of procedures to identify the place of beginning and end of all sections of the signal (Fig. 3). First, the signal is averaged using a moving average. This method is very well suited as the low-pass filter removes high frequency components of the signal [19]. In the next

Table 1 Parameter settings for the basic test procedure.

\begin{tabular}{ll}
\hline Parameter & Parameter value \\
\hline Jaw width & $25 \mathrm{~mm}$ \\
Couch travel per gantry rotation & $10 \mathrm{~mm}$ \\
Couch velocity & $0.5 \mathrm{~mm} / \mathrm{s}$ \\
Duration of the procedure & $320 \mathrm{~s}$ \\
Time of gantry rotation & $20 \mathrm{~s}$ \\
Collimator MLC & Static \\
The total number of leaves of the MLC & 64 \\
The number of open leaves of the MLC & 20 middle leaves \\
\hline
\end{tabular}

section, the first derivative is generated for each sample and its zero point found allows to indicate the boundary points of segments. The last two modules included in the macros are responsible for the calculation of the parameters of all segments (i.e. duration, length, average signal value, standard deviation, flatness, the ratio of the average signal) and the calculation of the time of gantry rotation. Data are presented in the form of a final report and chart.

\section{Measured and calculated parameters}

A total of 18 basic test procedures were performed, on average 1 test per 2 days. The last 7 procedures were carried out after a general, annual review of the tomotherapy machine, during which the target was replaced, main jaws calibrated, and a series of tests performed to

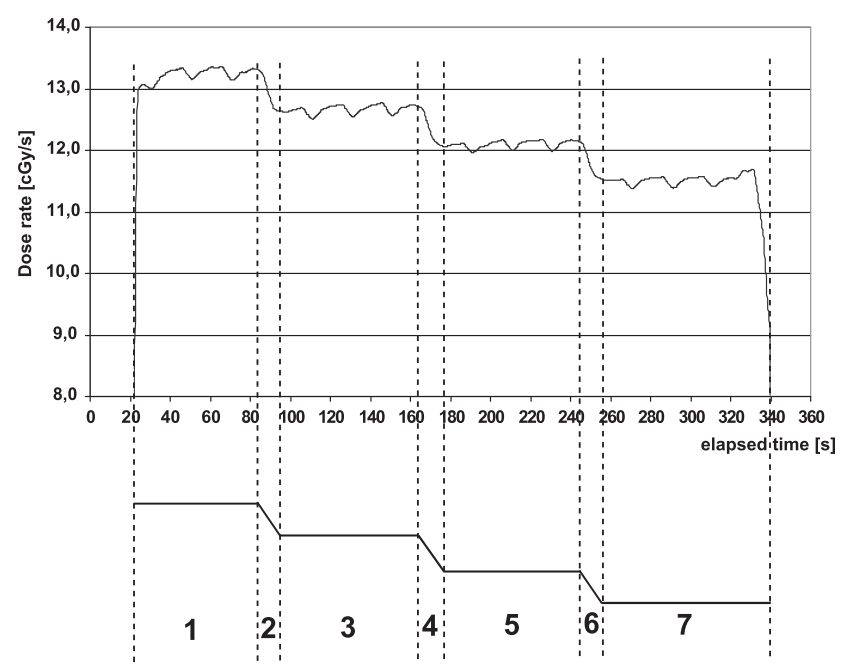

Figure 3 View of a sample signal obtained by the data acquisition system using TEMS (upper image) and schematic presentation of its various sections (lower image). 
verify correct operation of all elements of the machine. The parameters which were calculated and compared for each of the 18 signals include:

(a) Measurement of the couch velocity - calculated as the ratio of the fixed length of the partial cylinder of the length $40 \mathrm{~mm}$ to the duration of sections:

$t_{A}=t_{2}+t_{3}$

and

$t_{B}=t_{4}+t_{5}$,

where $t_{1}, t_{2}, t_{3}, t_{4}$ are the durations of adequate segments (Fig. 3).

(b) Measurement of the dose rate at depth - calculation of the average dose rate of $D_{1}, D_{3}, D_{5}, D_{7}$ for each flat sections of the resulting signal.

(c) Calculation of the coefficients Dose Ratio - calculation of the ratio of dose rates at appropriate depths, and thus, respectively:

- $\mathrm{DR}_{3-1}$ - the ratio of the dose rates of section 3 to section 1,

- $\mathrm{DR}_{5-1}$ - the ratio of the dose rates of section 5 to section 1,

- $\mathrm{DR}_{7-1}-$ the ratio of the dose rates of section 7 to section 1.

(d) Calculation of a coefficient of dose rate variation, for the value of step (b) (so-called: Dose Flatness) - to determine the magnitude of changes in dose rate, which fluctuates around the mean value. The coefficient is determined by the ratio:

$\mathrm{DF}_{i}=\frac{S_{i}}{D_{i}}$

for $i=1,3,5,7$, where $S_{i}$ is the standard deviation for the dose rate of the $i$ th segment.

(e) The calculation of the time of gantry rotation $T_{G R}-$ a calculation based on the analysis of periodic dose rate changes for sections 3 and 5 . The calculation based on the effect of Variation of Rotation measured as the time between successive extremes of the signal.

\section{Results}

\section{Couch velocity}

Appropriate formulas Equation (1) and Equation (2) allowed to calculate the velocity of the couch for two equal length consecutive sections of the couch - section 2 and $3(A)$ and section 3 and 4 (B) - for each procedure separately. Fig. 4 shows a graph of changes in speed $V_{A}$ and $V_{B}$ at the time. The calculated values deviate much less from the expected value, equal to $0.500 \mathrm{~mm} / \mathrm{s}$, for procedures performed after review of the machine. To verify the existence of differences between the $V_{A}$ and $V_{B}$ before and after the review, the speed values were separated into four groups: group 1 and 2 containing the velocity $V_{A}$ and group 3 and 4

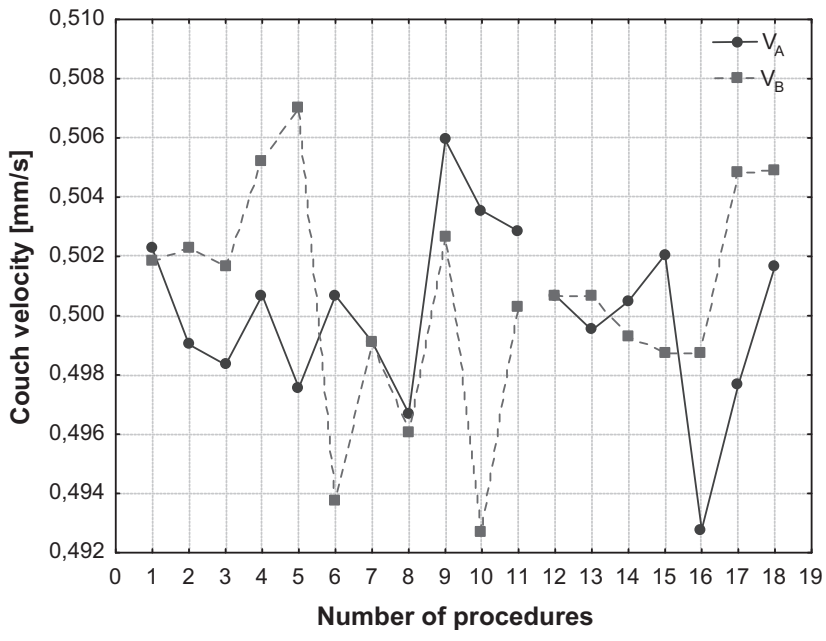

Figure 4 The graph of speed changes $V_{A}$ and $V_{B}$ couch travel.

containing the velocity $V_{B}$, obtained before and after review of the machine, respectively.

Table 2 presents the descriptive statistics of individual groups. In each of them, the distribution of results does not differ from the normal distribution and the data show homogeneity of variance. The test analysis of variance (ANOVA) showed no statistically significant differences in the speed between groups (probability value $p \leq 0.777$ ). At the end, calculations were made to obtain the mean value and standard deviation of the couch velocity of $0.500 \pm 0.003[\mathrm{~mm} / \mathrm{s}]$ from all 36 measurements, which is consistent with the set value for the irradiation procedure.

Twice the standard deviation, which applies to $95 \%$ of all the results, provides a reference interval. It is equal to $0.006[\mathrm{~mm} / \mathrm{s}]$, which represents $1.2 \%$ of the reference value. In summary, we can conclude that the measuring system used can calculate the speed of the table with an accuracy of $0.001[\mathrm{~mm} / \mathrm{s}]$ and the calculated value should not exceed the range of $\pm 1.2 \%$ reference value. Exceeding this range may indicate a malfunction of the machine. This is a very precise method. For comparison, other tests $[4,11,14]$ provide tolerance of $\pm 2 \%$.

\section{The value of the dose rate at the depth}

The average value of the signal for sections $1,3,5$ and 7 (Fig. 3) allowed to obtain information about the radiation dose rate at different depths of the phantom $-14.75 \mathrm{~mm}$, $24.75 \mathrm{~mm}, 34.75 \mathrm{~mm}, 44.75 \mathrm{~mm}$, respectively. Fig. 5 shows how the values of these dose rates slightly differ for

Table 2 Summary of descriptive statistics for the couch velocity per group.

\begin{tabular}{llcl}
\hline Group & $\begin{array}{l}\text { Mean couch } \\
\text { velocity value } \\
{[\mathrm{mm} / \mathrm{s}]}\end{array}$ & $\begin{array}{l}\text { Number of } \\
\text { samples }\end{array}$ & $\begin{array}{l}\text { Std. Dev. } \\
{[\mathrm{mm} / \mathrm{s}]}\end{array}$ \\
\hline 1 & 0.5006 & 11 & 0.0028 \\
2 & 0.4992 & 7 & 0.0032 \\
3 & 0.5002 & 11 & 0.0045 \\
4 & 0.5011 & 7 & 0.0027 \\
\hline
\end{tabular}




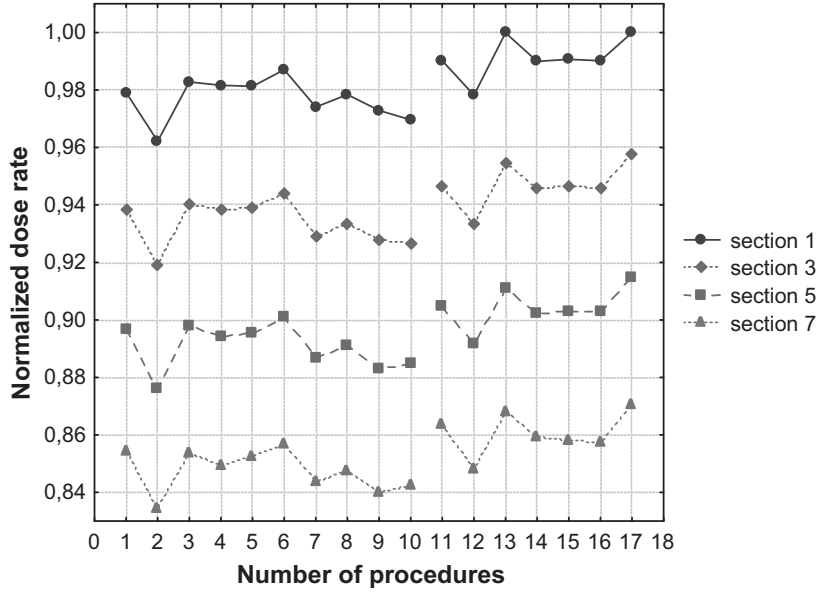

Figure 5 The graph of changes of the average dose rate value in time, for four different depths. Data were normalized to the maximum dose rate value obtained from all procedures.

different procedures. Data were normalized to the maximum dose rate value obtained from all procedures (the maximum value was obtained for the 17 th procedure). Equal dose rate verification was done before and after the review of machine. Table 3 contains descriptive statistics (average dose rate, standard deviation) for all sections, including the breakdown into those with measurement made before and after the review of the machine. Here we compare two mean values for 4 different cases (4 sections). $T$-test for unpaired variables showed a statistically significant difference between the dose measured before and after the review of the machine. Hence, it was decided to take mean values of dose rate calculated after the review of the machine as reference values (probability value for the section 1: $p \leq 0.0011 ; 3: p \leq 0.0032 ; 5: p \leq 0.0023 ; 7$ : $p \leq 0.0022$ ). The reference intervals were defined by twice the standard deviation. In relation to the dose rate, it is an interval of $\pm 1.8 \%$ for each tested depth.

\section{Dose ratio coefficients}

Based on the data with values of dose rate at depth, their ratios were calculated to yield values of $\mathrm{DR}_{3-1}, \mathrm{DR}_{5-1}$, and $D R_{-1}$. The results are very well illustrated in Fig. 6. To make sure that the values of DR did not differ significantly before and after the review of the machine, the results were separated into 6 groups (each type of DR, is assigned a group of results obtained before and after the review). Table 3 presents descriptive statistics for each group. In each of these, the distribution of data does not differ from normal, and the $t$-test for unpaired variables showed no statistically significant difference in the values of Dose Ratio before and after the review of the machine (the probability value for $\mathrm{DR}_{3-1}: p \leq 0.620 ; \mathrm{DR}_{5-1}: p \leq 0.625$ and $\left.\mathrm{DR}_{7-1}: p \leq 0.554\right)$. Therefore, mean values and standard deviations were calculated for the total number of procedures (18 procedures), which are (Mean \pm SD):

- for $\mathrm{DR}_{3-1}: 0.9557 \pm 0.0013$

- for $\mathrm{DR}_{5-1}: 0.9121 \pm 0.0018$

- for $\mathrm{DR}_{7-1}: 0.8679 \pm 0.0023$

Table 3 The descriptive statistics of dose rates at depth, Dose Ratio and Dose Flatness coefficients, including the division into groups: before and after the review of the machine.

\begin{tabular}{|c|c|c|c|c|c|}
\hline Parameter & $\begin{array}{l}\text { Section/ } \\
\text { coefficient }\end{array}$ & $\begin{array}{l}\text { Execution time } \\
\text { before/after } \\
\text { review }\end{array}$ & $\begin{array}{l}\text { Number of } \\
\text { samples }\end{array}$ & $\begin{array}{l}\text { Average value of } \\
\text { parameter }\end{array}$ & Std. Dev. \\
\hline \multirow{8}{*}{$\begin{array}{l}\text { Dose rate } \\
\text { value at a } \\
\text { depth }\end{array}$} & 1 & Before & 10 & $13.1[\mathrm{cGy} / \mathrm{s}]$ & $0.1[\mathrm{cGy} / \mathrm{s}]$ \\
\hline & 1 & After & 7 & $13.3[\mathrm{cGy} / \mathrm{s}]$ & $0.1[\mathrm{cGy} / \mathrm{s}]$ \\
\hline & 3 & Before & 10 & $12.5[\mathrm{cGy} / \mathrm{s}]$ & $0.1[\mathrm{cGy} / \mathrm{s}]$ \\
\hline & 3 & After & 7 & $12.7[\mathrm{cGy} / \mathrm{s}]$ & $0.1[\mathrm{cGy} / \mathrm{s}]$ \\
\hline & 5 & Before & 10 & $12.0[\mathrm{cGy} / \mathrm{s}]$ & $0.1[\mathrm{cGy} / \mathrm{s}]$ \\
\hline & 5 & After & 7 & $12.1[\mathrm{cGy} / \mathrm{s}]$ & $0.1[\mathrm{cGy} / \mathrm{s}]$ \\
\hline & 7 & Before & 10 & $11.3[\mathrm{cGy} / \mathrm{s}]$ & $0.1[\mathrm{cGy} / \mathrm{s}]$ \\
\hline & 7 & After & 7 & $11.6[\mathrm{cGy} / \mathrm{s}]$ & $0.1[\mathrm{cGy} / \mathrm{s}]$ \\
\hline \multirow[t]{6}{*}{ Dose Ratio } & $\mathrm{DR}_{3-1}$ & Before & 11 & 0.9558 & 0.0015 \\
\hline & $\mathrm{DR}_{3-1}$ & After & 7 & 0.9555 & 0.0010 \\
\hline & $\mathrm{DR}_{5-1}$ & Before & 11 & 0.9119 & 0.0021 \\
\hline & $\mathrm{DR}_{5-1}$ & After & 7 & 0.9123 & 0.0012 \\
\hline & $\mathrm{DR}_{7-1}$ & Before & 11 & 0.8676 & 0.0024 \\
\hline & $\mathrm{DR}_{7-1}$ & After & 7 & 0.8623 & 0.0023 \\
\hline \multirow[t]{8}{*}{ Dose Flatness } & $\mathrm{DF}_{1}$ & Before & 11 & 0.0058 & 0.0006 \\
\hline & $\mathrm{DF}_{1}$ & After & 7 & 0.0058 & 0.0005 \\
\hline & $\mathrm{DF}_{3}$ & Before & 11 & 0.0052 & 0.0009 \\
\hline & $\mathrm{DF}_{3}$ & After & 7 & 0.0060 & 0.0005 \\
\hline & $\mathrm{DF}_{5}$ & Before & 11 & 0.0049 & 0.0010 \\
\hline & $\mathrm{DF}_{5}$ & After & 7 & 0.0058 & 0.0005 \\
\hline & $\mathrm{DF}_{7}$ & Before & 11 & 0.0046 & 0.0010 \\
\hline & $\mathrm{DF}_{7}$ & After & 7 & 0.0060 & 0.0005 \\
\hline
\end{tabular}




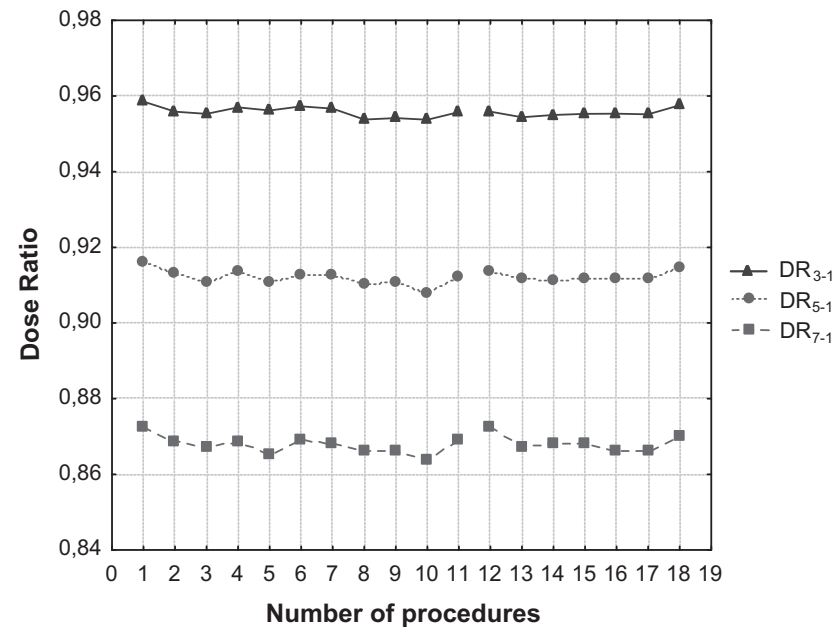

Figure 6 The graph of changes in the coefficients Dose Ratio in time.

At the end, reference intervals were estimated defined as twice the level of largest standard deviation, which makes the value of $\pm 0.5 \%$ for the Dose Ratio for each factor.

\section{Dose flatness coefficients}

The mean values and standard deviations for the dose rates at depth were used to calculate Dose Flatness coefficients. In the case of measurements that were performed before the review of the machine, coefficients had lower values and a large dispersion (Fig. 7). In order to test the differences between the values of Dose Flatness for each section, a table was made containing descriptive statistics for each of them (Table 3 ). To compare the differences in average values, it was decided to use the analysis of variance (ANOVA). Distribution of results for each group did not deviate from normal and the Brown-Forsythe test showed homogeneity of variance. Results of the analysis of variance showed a statistically

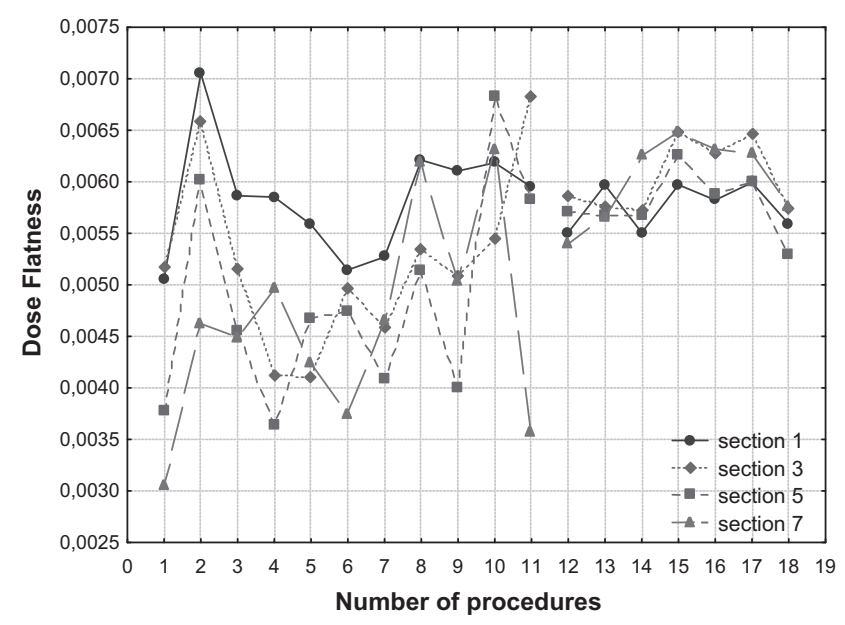

Figure 7 The graph of changes in time for the Dose Flatness coefficients. significant difference between average values in the groups ( $p \leq 0.001$ ). A post-hoc Duncan's test showed the differences in average values of the Dose Flatness coefficients only for the comparisons between any two groups of measurements taken before and after the review of the machine. This results could be expected after the analysis of dose rates at depth.

In the case of coefficients of variation DF, it is impossible to determine the average value that will be considered as a reference. They depend on the dose rate at the depth and standard deviation (amplitude of signal). The range of values for Dose Flatness for measurements taken after the review is: $\mathrm{DF}_{\min }=0.0053, \mathrm{DF}_{\max }=0.0065$. We can then determine the possible percentage range for the value of $D F$, i.e. $D F=(0.53-0.65) \%$.

\section{Time of gantry rotation}

The algorithm of macros of the stepwedge Analysis allows to calculate the time of gantry rotation from the analysis of local extremes of the averaged signal. These times were calculated for both the 3 rd and the 5 th section of the signal. The resulting data is illustrated in Fig. 8. The significant dispersion of results obtained before the review of the machine is noticeable. The collected data were divided due to the time of measurement. They were separated into 2 groups of measurements taken before the review of the machine:

- group A - the average time of gantry rotation for the 3rd section,

- group $B$ - the average time of gantry rotation for the 5th section,

and two groups for measurements performed after the review:

- group $\mathrm{C}$ - the average time of gantry rotation for the 3rd section,

- group $\mathrm{D}$ - the average time of gantry rotation for the 5 th section.

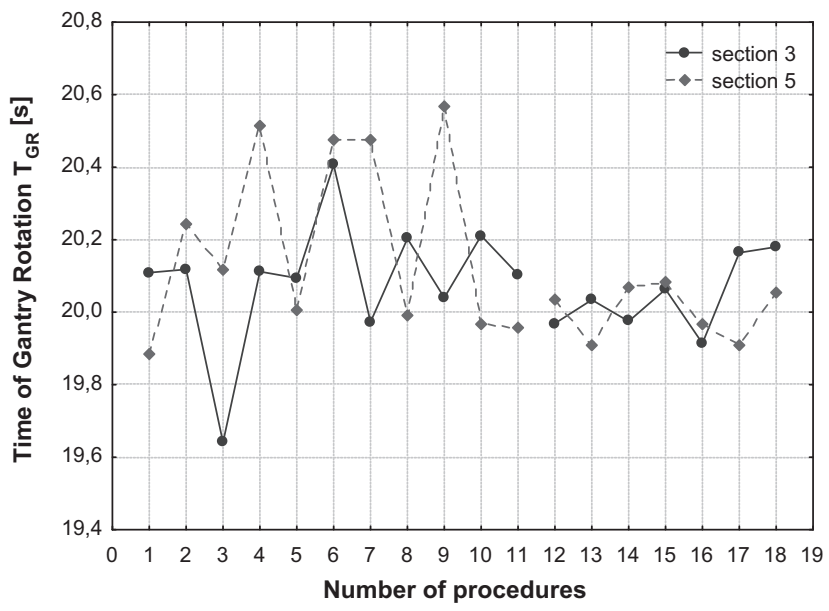

Figure 8 The graph of changes in the time of gantry rotation in time for the two analyzed sections. 
Table 4 Summary of descriptive statistics for the time of gantry rotation per group.

\begin{tabular}{llll}
\hline Group & $\begin{array}{l}\text { Average time of } \\
\text { gantry rotation [s] }\end{array}$ & $\begin{array}{l}\text { Number of } \\
\text { samples }\end{array}$ & Std. Dev. [s] \\
\hline A & 20.082 & 42 & 0.458 \\
B & 20.049 & 27 & 0.357 \\
C & 20.187 & 49 & 0.464 \\
D & 20.014 & 24 & 0.340 \\
\hline
\end{tabular}

Descriptive statistics were determined for each group (Table 4). It allowed in the next step to verify the existence of differences in the average time of gantry rotation between the groups. After successfully passing the test for homogeneity of variance and normality of distribution, the test of analysis of variance (ANOVA) was performed for unpaired variables. It did not show statistically significant differences in average values of the time (probability value $p \leq 0.18$ ). A $t$-test was also made to verify the compliance of the average time of gantry rotation for each group with an expected value of $20 \mathrm{~s}$. The probabilities for particular groups were: group $\mathrm{A}: p \leq 0.251$; group $\mathrm{B}: p \leq 0.487$; group C: $p \leq 0.007$; group $D: p \leq 0.837$. As a result of the test, samples from Group $C$ were eliminated and the remaining values were used to calculate the average rotation time of the probe and the standard deviation (a total of 93 samples). These are: $20.055 \pm 0.302$ [s].

Twice the standard deviation gives us a reference interval. It is equal to 0.602 [s] which corresponds to about $3 \%$ of the reference value. Thus, the time value of gantry rotation measured with a macro should be between 19.4 and 20.6 [s]. There is a noticeable spread of results and thus low accuracy of the method of detection, because the difference of $0.6[\mathrm{~s}]$ in the time of gantry rotation, as a systematic error, is an unacceptable value in the further work of tomotherapy machine. Another method of detection of gantry rotation time should be considered based on the oscillation signal value. Unfortunately, it should be noted that with increasing accuracy of detection the number of samples taken to measure decreases, and the other way round.

\section{Conclusion}

The developed the cylindrical stepwedge phantom allows with high precision to determine the couch velocity, the dose rate at depth, the Dose Ratio, the Dose Flatness coefficients and the time of gantry rotation for a tomotherapy machine during a single irradiation procedure (lasting $5 \mathrm{~min}$ ). In addition measurements are made independently of the machine. It is a good alternative for some commonly used QA tests.

In this study critical values of parameter ranges have been set in the form of rates and maximum deviations from the reference values. This confirms the high accuracy of the measured parameters, which in the case of other tests is usually in the range of $\pm 2 \%$. For the values of gantry rotation time, we achieved the accuracy of $\pm 3 \%$, which is not acceptable for the tomotherapy machine. Although, the idea of measurement is correct, the way of signal analysis should be changed, which will surely increase the precision of the time determination.

\section{References}

[1] Balog J, Mackie TR, Reckwerdt P, Glass M, Angelos L. Characterization of the output for helical delivery of intensity modulated slitbeams. Med Phys 1999;26:55-64.

[2] Balog J, Mackie TR, Pearson D, Hui S, Paliwal B, Jeraj R. Benchmarking beam alignment for a clinical helical tomotherapy device. Med Phys 2003;30:1118-27.

[3] Balog J, Olivera G, Kapatoes J. Clinical helical tomotherapy commissioning dosimetry. Med Phys 2003;30:3097-106.

[4] Langen KM, Papanikolaou N, Chngyu S, Balog J, Crilly R, Followil D, et al. QA for helical tomotherapy: report of the AAPM Task group 148. Med Phys 2010;37:4817-54.

[5] Bhardwaj AK, Sharma SC, Rana B, Shukla A. Study of 2D ion chamber array for angular response and QA of dynamic MLC and pretreatment IMRT plans. Rep Pract Oncol Radiother 2009;14(3):89-94.

[6] Kutcher GJ, Coia L, Gillin M, Hanson WF, Leibel S, Morton RJ, et al. Comprehensive QA for radiation oncology: report of AAPM radiation therapy Committee Task group 40. Med Phys 1994;21:581-618.

[7] Low DA, Mutic S, Dempsey JF, Gerber RL, Bosch WR, Perez CA, et al. Quantitative dosimetric verification of an IMRT planning and delivery system. Radiother Oncol 1998;49:305-16.

[8] Malicki J, Litoborski M, Bogusz-Czerniewicz M, Świeżewski A. Cost-effectiveness of the modifications in the quality assurance system in radiotherapy in the example of in-vivo dosimetry. Phys Med 2009;25:201-6.

[9] Woo SY, Grant W, McGary JE, Teh BS, Butler EB. The evolution of quality assurance for intensity modulated radiation therapy (IMRT): sequential tomotherapy. Int J Radiat Oncol Biol Phys 2003;56:274-86.

[10] Sukumar P, Padmanaban S, Jeevanandam P, Syamkumar SA, Nagarajan V. A study on dosimetric properties of electronic portal imaging device and its use as a quality assurance tool in Volumetric Modulated Arc Therapy. Rep Pract Oncol Radiother 2011;16(6):248-55.

[11] TomoTherapy Incorporated. Tomotherapy HiArt system${ }^{\circledR}$ physics verification; 2007.

[12] Wong JH, Hardcastle N, Tomé WA, Bayliss A, Tolakanahalli R, Lerch ML, et al. Independent quality assurance of a helical tomotherapy machine using the dose magnifying glass. Med Phys 2011;38(4):2256-64.

[13] Mackie TR, Holmes T, Swerdloff S, Reckwerdt P, Deasy JO, Yang J, et al. Tomotherapy: a new concept for the delivery of dynamic conformal radiotherapy. Med Phys 1994;20:1709-19.

[14] Fenwick JD, Tome WA, Jaradat HA, Hui SK, James JA, Balog JP, et al. Quality assurance of a helical tomotherapy machine. Phys Med Biol 2004;49:2933-53.

[15] Balog J, Holmes T, Vaden R. Helical tomotherapy dynamic quality assurance. Phys Med 2006;33(10):3939-50.

[16] Bindhu J, Supe S, Pawar Y. Intensity modulated radiotherapy (IMRT) the white, black and grey: a clinical perspective. Rep Pract Oncol Radiother 2009;14(3):95-103.

[17] Malicki J, Maciejewski B, Ślosarek K, Drzewiecka B. Radiobiological rationale for advantages and limitations of IMRT in clinical practice. Nowotwory 2001;51:355-64.

[18] Ślosarek K, Grzadziel A, Osewski W, Dolla $Ł$, Bekman B, Petrovic $B$. Beam rate influence on dose distribution and fluence map in IMRT dynamic technique. Rep Pract Oncol Radiother 2012, in press.

[19] Smith SW. Digital signal processing. A Pract Guide for Engineers Scientists 2003;15:277-84. 